\title{
FKBP5 Moderates the Association between Antenatal Maternal Depressive Symptoms and Neonatal Brain Morphology
}

\author{
Changqing Wang', Mojun Shen ${ }^{2}$, Bryan Guillaume', Yap-Seng Chong ${ }^{2,3}$, Helen Chen ${ }^{4}$, Marielle V Fortier, \\ Michael J Meaney ${ }^{2,6,7}$ and Anqi Qiu*,1,2 \\ 'Department of Biomedical Engineering and Clinical Imaging Research Center, National University of Singapore, Singapore; '2 Singapore Institute for \\ Clinical Sciences, Singapore; '3 Department of Obstetrics and Gynaecology, Yong Loo Lin School of Medicine, National University of Singapore, \\ National University Health System, Singapore; 'KK Women's and Children's Hospital, Duke-National University of Singapore, Singapore; \\ ${ }^{5}$ Department of Diagnostic and Interventional Imaging, KK Women's and Children's Hospital (KKH), Singapore; 'Ludmer Centre for \\ Neuroinformatics and Mental Health, Douglas Mental Health University Institute, McGill University, Montreal, QC, Canada; ${ }^{7}$ Sackler Program for \\ Epigenetics and Psychobiology at McGill University, Montreal, QC, Canada
}

Antenatal maternal depressive symptoms influence fetal brain development and increase the risk for depression in offspring. Such vulnerability is often moderated by the offspring's genetic variants. This study aimed to examine whether FKBP5, a key regulator of the hypothalamic-pituitary-adrenal (HPA) axis, moderates the association between antenatal maternal depressive symptoms and in utero brain development, using an Asian cohort with 161 mother-offspring dyads. Antenatal maternal depressive symptoms were measured using the Edinburgh Postnatal Depression Scale (EPDS) during the second trimester of pregnancy. Neonatal structural brain images were acquired using magnetic resonance imaging (MRI) shortly after birth. Maternal and neonatal FKBP5 gene was genotyped using Illumina OmniExpress arrays. A gene set-based mixed effect model for gene-environment interaction (MixGE) was used to examine interactive effects between neonatal genetic variants of FKBP5 and antenatal maternal depressive symptoms on neonatal amygdala and hippocampal volumes, and cortical thickness. Our study revealed that genetic variants in neonatal FKBP5 moderate the association between antenatal maternal depressive symptoms and right hippocampal volume but only show a trend for such moderation on amygdala volumes and cortical thickness. Our findings are the first to reveal that the association between maternal depressive symptoms and in utero neurodevelopment of specific brain regions is modified through complex genetic variation in neonatal FKBP5. Our results suggest that an increased risk for depression may be transmitted from mother to child during fetal life and that the effect is dependent upon neonatal FKBP5 genotype. Neuropsychopharmacology (2018) 43, 564-570; doi:I0.1038/npp.2017.232; published online I November 2017

\section{INTRODUCTION}

Depression has a strong familial component. Children of mothers depressed during pregnancy show an increased risk for emotional, behavioral, and cognitive problems (Hammen and Brennan, 2003; Klein et al, 2001; Weissman et al, 2006), as well as multiple forms of psychopathology (Hammen and Brennan, 2003; Klein et al, 2001; Weissman et al, 2006) relative to the normal population. Similarly, increasing evidence suggests that fetal exposure to maternal depression influences intermediate phenotypes that are associated with vulnerability for depression (Feldman et al, 2009; Wichers et al, 2007), including alterations in brain morphology (Chen et al, 2010b; Peterson and Weissman, 2011). Importantly, antenatal maternal depression is associated with fetal brain

* Correspondence: Professor A Qiu, Department of Biomedical Engineering, National University of Singapore, 4 Engineering Drive 3, Engineering Block 4, 04-08, Singapore 1 17583, Singapore, Tel: +65 65167002, Fax: +65 65161516, E-mail: bieqa@nus.edu.sg

Received 13 June 2017; revised 21 August 2017; accepted 19 September 2017; accepted article preview online 3 October 2017 development, particularly in the amygdala and hippocampus, that are implicated in the regulation of emotional states (Qiu et al, 2013, 2015a). These effects appear to reflect the transmission of individual differences in vulnerability for depression from mother to child.

Vulnerability for depression in children of affected mothers may be moderated by genetic variants (Baumann et al, 2013). The FK506 binding protein 5 (FKBP5) encoded by the FKBP5 gene located on chromosome 6 is involved in hypothalamic-pituitary-adrenal (HPA) axis regulation. FKBP5 participates in the inhibition of glucocorticoid receptor (GR) activity, the main regulator of the HPA axis (Provencal and Binder, 2015). FKBP5 has been identified as a molecular genetic marker associated with depression (Binder et al, 2004; Zobel et al, 2010), posttraumatic stress disorder (PTSD) (Binder et al, 2008; Koenen and Uddin, 2010; Xie et al, 2010), and stress response (Velders et al, 2011). Carriers of the FKBP5 rs $1360780 \mathrm{~T}$ allele show an increased risk for depression (Lekman et al, 2008). Homozygotes of the rs1360780 $\mathrm{T}$ allele demonstrate more depressive episodes and better response to treatment with antidepressants 
(Binder et al, 2004). Similarly, high risk alleles of multiple FKBP5 single-nucleotide polymorphisms (SNPs) (rs3800373, rs9296158, rs1360780, rs7748266, and rs9470080) are associated with a decreased cortisol level and an increased risk of depressive symptoms (Velders et al, 2011). FKBP5 SNPs also moderate the impact of early-life stress that is linked with risk for later-life depression (Lahti et al, 2016).

Similarly, the function and microstructure of brain regions involved in emotion are influenced by early childhood trauma in the presence of FKBP5 risk alleles (Tozzi et al, 2016). Abused FKBP5 rs1360780 TT carriers show a significant reduction in hippocampal volume compared with abused CT/CC carriers (Grabe et al, 2016). High GR density and FKBP5 expression are observed in the hippocampus (Scharf et al, 2011). Animal research demonstrates that fetal exposure to a high level of glucocorticoids downregulates hippocampal GR expression (Levitt et al, 1996). This, coupled with the role of FKBP5 as a GR inhibitor, leads to symptoms of glucocorticoid resistance. Hence, these findings suggest a possible role of FKBP5 in moderating the influence of maternal depressive symptoms on fetal hippocampal development.

A prospective longitudinal mother-offspring cohort study (Growing Up in Singapore Towards Healthy Outcomes (GUSTO)) provided a unique opportunity to examine whether neonatal FKBP5 genotype moderates the association between antenatal maternal depressive symptoms and in utero hippocampal development without compromise from postnatal confounds. Given the link between generic variants of multiple FKBP5 SNPs and a risk of depression, a gene setbased mixed effect model for gene-environment interaction (MixGE) (Wang et al, 2017) was employed to test for interactive effect between neonatal genetic variants of FKBP5 and antenatal maternal depressive symptoms on hippocampal volume. Rather than testing each SNP individually, the MixGE model takes a set of SNPs and examines not only accumulative but also heterogeneous interactive effects of these individual SNPs with antenatal maternal depressive symptoms on neonatal hippocampal volumes. The MixGE model can detect both rare genetic variants and common genetic variants with small effect sizes by accumulating their effects within the genetic set, and by greatly reducing the number of independent statistical tests, and hence a less stringent correction for multiple comparisons is required (Lee et al, 2014). We hypothesized that FKBP5 genotype moderates the association between antenatal maternal depressive symptoms and neonatal hippocampal volumes. As an exploratory analysis, we also investigated whether FKBP5 moderates the association between antenatal maternal depression and in utero amygdala and cortical development. This study provides, to our knowledge, the first direct analysis of the genetic and environmental interactions associated with neonatal brain morphology and evidence that FKBP5 may represent a molecular genetic marker moderating differential sensitivity to antenatal maternal depression.

\section{MATERIALS AND METHODS}

\section{Subjects}

Mother-offspring dyads who participated in this imaging genetic study were part of the GUSTO cohort (Soh et al, 2012). The GUSTO cohort consisted of pregnant Asian women attending the first trimester antenatal ultrasound scan clinic at the National University Hospital (NUH) and KK Women's and Children's Hospital (KKH) in Singapore. The parents were Singapore citizens or Permanent Residents of Chinese, Malay, or Indian ethnic background. Birth outcome and pregnancy measures were obtained from hospital records. Socioeconomic status (household income) was extracted from survey questionnaires during pregnancy. Of the 184 mother-offspring dyads with neonatal neuroimaging data, those without genetic data $(n=9)$, antenatal maternal depression scores $(n=10)$, or demographic information $(n=3)$ were removed, leaving 164 motheroffspring dyads. Among these 164 mother-offspring dyads, neonates with gestational age $<34$ weeks $(n=0)$, birth weight $<2000 \mathrm{~g}(n=0)$, and a $5 \mathrm{~min}$, Appearance, Pulse, Grimace, Activity, and Respiration (APGAR) score $<9$ $(n=3)$ were removed, resulting in 161 mother-offspring dyads as the final sample for this study.

The GUSTO cohort study was approved by the National Healthcare Group Domain Specific Review Board (NHG DSRB) and the Sing Health Centralized Institutional Review Board (CIRB). Written informed consent was obtained from mothers.

\section{Antenatal Maternal Depressive Symptoms}

The Edinburgh Postnatal Depression Scale (EPDS) questionnaire (Cox et al, 1987) was administered to mothers at 26 weeks of pregnancy to quantify depressive symptomatology. The EPDS is a widely used 10 -item self-report scale designed as a screening instrument for postnatal depression, but it is also valid for use during pregnancy. Each item of the EPDS is scored on a four-point scale (0-3), for a total score of 30. A higher total score indicates presence of more depressive symptoms. The reliability of the EPDS for our cohort assessed using Cronbach's analysis is 0.82 .

\section{Magnetic Resonance Imaging (MRI) Acquisition and Analysis}

Axial fast spin-echo T2-weighted MRI (repetition time $(\mathrm{TR})=3500 \mathrm{~ms}$; echo time $(\mathrm{TE})=110 \mathrm{~ms}$; field of view $($ FOV $)=256 \mathrm{~mm} \times 256 \mathrm{~mm}$; matrix size $=256 \times 256$; 50 axial slices with $2.0 \mathrm{~mm}$ thickness) were acquired for neonates at 5 to 14 days of age using a 1.5-Tesla GE scanner at the Department of Diagnostic and Interventional Imaging of the KK Women's and Children's Hospital. Detailed acquisition and image quality check procedures have been previously reported (Qiu et al, 2013; Qiu et al, 2015b). All brain data were acquired when neonates were in sleep after feeding. A sip of glucose was given if neonates woke up in the scanner. The scanning was restarted after neonates fell into sleep. No sedation was used and precautions were taken to reduce exposure of neonates to MRI scanner noise. A neonatologist was present during scanning. A pulse oximeter was used to monitor heart rate and oxygen saturation throughout entire 
scans. All brain scans were reviewed by a neuroradiologist. To determine image motion, all axial slices of the T2weighted MRI data were visually inspected to ensure no cross-slice motion and checkerboard patterns.

Within individual subjects, a Markov random field model (MRF) was used to automatically delineate amygdala, hippocampus, cortical gray matter, white matter, and cerebrospinal fluid (CSF) from the neonatal T2-weighted MRI data. Manual segmentation was performed on 20 brains randomly selected from our data set. The segmentation accuracy rates of the amygdala, hippocampus, cortical gray matter, and white matter of the automatic segmentation against the manual segmentation were $0.75,0.71,0.79$, and 0.86 , respectively. This has also been reported in previous publications (Qiu et al, 2013, 2015b; Rifkin-Graboi et al, 2013).

Based on the above tissue segmentation, cortical surfaces were constructed at the boundary between gray matter and CSF and at the boundary of gray matter and white matter, using a graph-based topology correction algorithm. Cortical thickness was measured as the distances between the corresponding vertices of these two cortical surfaces. We employed a large deformation diffeomorphic metric mapping (LDDMM) algorithm to align individual cortical surfaces to the Asian atlas that was generated based on the cortical anatomy of the 20 subjects with manual segmentation, and transferred the thickness values of each subject to the atlas (Bai et al, 2012). The cortical thickness values were smoothed using the Laplace-Beltrami basis functions on the cortical surface.

\section{SNP Genotyping}

Genomic DNA was extracted from frozen umbilical cord specimens for offspring and from blood for mothers per standard procedures. The samples were then genotyped on both Illumina OmniExpress arrays, which perform well and have better coverage than competitors in Asian populations (Jiang et al, 2013), and on Illumina Exome arrays, following the manufacturer's instructions by Expression Analysis. Data were processed in GenomeStudio Genotyping Module. Genotyping calls were made by the GenCall software that incorporates a clustering algorithm (GenTrain) and a calling algorithm (Bayesian model). The GenCall Score of each SNP probe and call rate of each sample were generated. The GenCall score is primarily designed as a means by which to rank and filter failed genotypes (Oliphant et al, 2002). Scores of $<0.15$ generally indicate failed genotypes and hence the genotypes with a GenCall score of $<0.15$ are not assigned genotypes (Oliphant et al, 2002). Total 30 SNPs of FKBP5 gene (Chr 6: 35540362-35697360) were extracted on these two arrays. Among them, 2 SNPs did not pass the GenCall score and 9 SNPs were homogeneous among our subjects. The remaining 19 SNPs were extracted and used in this study as a gene set. Table 2 and Supplementary Table S1 (the Supplementary Material) list the genotype distributions of these SNPs for neonates and mothers included in this study. As the statistical model described below was designed to incorporate rare alleles, rs16879378 with minimum allele frequency (MAF) of 0.006 was also included in this study. The 19 FKBP5 SNPs did not deviate from Hardy-Weinberg proportions after correction for multiple comparisons. No imputation was applied to the genetic data.

\section{Statistical Analysis}

The aim of this study was to examine interactive effects between genetic variants of neonatal FKBP5 with antenatal maternal depressive symptoms on neonatal hippocampal volumes. A gene set-based mixed effect model for MixGE was employed (Wang et al, 2017). The software and example are available at http://www.bioeng.nus.edu.sg/cfa/imagingge netics.html. The MixGE model is in the form of

$Y=Z \beta+\operatorname{diag}(E) G \mathbf{1} \pi+\operatorname{diag}(E) G \delta$

where $Z=[X, E, G]$, where $X$ consists of gestational age on the MRI visit day, neonatal total brain volume, maternal education, gender, and maternal ethnicity. $Y$ is the hippocampal volume, $E$ is the score of antenatal maternal depressive symptoms, and $G$ represents a set of the 19 SNPs of neonatal FKBP5. The diag (E) $G$ therefore represents gene-environment interaction $(G \times$ E). 1 represents a vector of ones of length 19. The $\pi$ is a fixed effect of $\mathrm{G} \times \mathrm{E}$ and $\delta$ is random effects of $\mathrm{G} \times \mathrm{E}$. The $\pi$ captures the accumulative $\mathrm{G} \times \mathrm{E}$ effects, whereas $\delta$ captures the heterogeneous $\mathrm{G} \times \mathrm{E}$ effects among all the 19 SNPs. As the genetic variants are considered as a set rather than individually, the $G \times E$ effects of both rare genetic variants and common genetic variants with small effect sizes can be detected, as their effects are accumulated within the genetic set. Furthermore, by greatly reducing the number of independent statistical tests, a less stringent correction for multiple comparisons is required (Lee et al, 2014).

The analysis using the MixGE model was repeated when considering maternal variants of FKBP5 and its interaction with antenatal maternal depressive symptoms as additional covariates. In other words, $Z=\left[X, E, G, G_{\mathrm{M}}, \operatorname{diag}(E) G_{\mathrm{M}} \mathbf{1}\right]$, where $G_{\mathrm{M}}$ are the 19 SNPs of maternal FKBP5.

Regression analysis was also performed to examine interaction between individual neonatal FKBP5 SNPs and antenatal maternal depressive symptoms on neonatal hippocampal volumes after partialling out the covariance of gestational age on the MRI visit day, neonatal total brain volume, maternal education, gender, and maternal ethnicity. This analysis was performed for 17 of the 19 SNPs of FKBP5 with $\mathrm{MAF}>0.05$.

The same statistical analysis was applied to amygdala volume and cortical thickness. Bonferroni correction was employed for bilateral hippocampal and amygdala volumes $(p<0.05 / 4=0.0125)$. For cortical thickness, the $p$-values of the 112200 vertices in both hemispheres were corrected via false discovery rate (FDR).

\section{RESULTS}

Table 1 lists the demographics of the 161 mother-offspring dyads in this study. Gestational age at birth $\left(t_{159}=-0.718\right.$, $p=0.474)$, birth weight $\left(t_{159}=-0.679, p=0.498\right)$, gestational age on the MRI visit day $\left(t_{159}=-0.505, p=0.614\right)$, neonatal total brain volume $\left(t_{159}=-0.387, p=0.699\right)$, and maternal education $\left(t_{159}=-1.51, p=0.134\right)$ did not vary as a function of antenatal maternal depressive symptoms. Difference in antenatal maternal depressive symptoms among maternal ethnic groups was marginally significant $\left(\mathrm{F}_{2,158}=3.02\right.$, 
Table I Demographics and Brain Volumes

\begin{tabular}{|c|c|c|c|}
\hline & & Mean & SD \\
\hline \multicolumn{2}{|l|}{ Gestational age at birth (weeks) } & 38.9 & l. \\
\hline \multicolumn{2}{|l|}{ Birth weight (kg) } & 3.11 & 0.39 \\
\hline \multicolumn{2}{|l|}{5 min APGAR score } & 9.01 & 0.08 \\
\hline \multicolumn{2}{|l|}{ Gestational age at MRI (weeks) } & 40.3 & l. 1 \\
\hline \multicolumn{2}{|l|}{ Antenatal maternal EPDS score } & 8.56 & 4.5 \\
\hline \multicolumn{2}{|l|}{ Neonatal total brain volume $\left(\mathrm{cm}^{3}\right)$} & 549 & 47 \\
\hline \multicolumn{2}{|l|}{ Neonatal left hippocampal volume $\left(\mathrm{mm}^{3}\right)$} & 779 & 107 \\
\hline \multicolumn{2}{|l|}{ Neonatal right hippocampal volume $\left(\mathrm{mm}^{3}\right)$} & 784 & $11 \mid$ \\
\hline \multicolumn{2}{|l|}{ Neonatal left amygdala volume $\left(\mathrm{mm}^{3}\right)$} & 211 & 35 \\
\hline \multirow{2}{*}{\multicolumn{2}{|c|}{ Neonatal right amygdala volume $\left(\mathrm{mm}^{3}\right)$}} & 187 & 34 \\
\hline & & $N$ & $\%$ \\
\hline \multirow[t]{5}{*}{ Maternal education } & Primary & 6 & 3.7 \\
\hline & Secondary & 54 & 33.5 \\
\hline & GCE/Diploma & 72 & 44.7 \\
\hline & Undergraduate & 23 & 14.3 \\
\hline & Graduate & 6 & 3.7 \\
\hline \multirow[t]{3}{*}{ Maternal ethnicity } & Chinese & 73 & 45.3 \\
\hline & Malay & 67 & 41.6 \\
\hline & Indian & 21 & 13 \\
\hline \multirow[t]{2}{*}{ Gender of neonates } & Male & 87 & 54 \\
\hline & Female & 74 & 46 \\
\hline \multirow[t]{5}{*}{ Monthly household } & income $<\$ 999$ & 4 & 2.4 \\
\hline & $\$ 1000-\$ 1999$ & 24 & 16 \\
\hline & $\$ 2000-\$ 3999$ & 60 & 40 \\
\hline & $\$ 4000-\$ 5999$ & 39 & 26 \\
\hline & $>\$ 6000$ & 23 & 15.3 \\
\hline Mothers on antidepressants & & 0 & 0 \\
\hline
\end{tabular}

$p=0.052)$. Monthly household income was highly correlated with antenatal maternal depressive symptoms $(\rho=-0.277$, $p<0.001)$, maternal education $(\rho=0.520, p<0.001)$, and significantly differed among maternal ethnic groups $\left(F_{2,147}=5.43, p=0.005\right)$. To avoid collinearity, the following analysis did not include monthly household income as covariate. Maternal education was chosen over monthly household income as it is a much more stable indicator of social economic status.

Table 2 and Supplementary Table S1 (the Supplementary Material) list the distributions of individual FKBP5 SNPs in the children and mothers. Antenatal maternal depressive symptoms were not associated with the neonatal $(p=0.729)$ or maternal $(p=0.855)$ FKBP5 genotype. Gestational age at birth $(p=0.190)$, birth weight $(p=0.984)$, and neonatal total brain volume $(p=0.368)$ were not associated with neonatal FKBP5 genotype.

The MixGE model revealed a significant interaction of neonatal FKBP5 and antenatal maternal depressive symptoms on the right $\left(p=5.68 \times 10^{-4}\right)$ but not on the left hippocampal volume $(p=0.095)$ after partialling out the covariance of gestational age on the MRI visit day, neonatal total brain volume, maternal education, gender, and maternal ethnicity. After additionally controlling for maternal FKBP5, the neonatal FKBP5 interaction with maternal depressive symptoms showed a marginally significant effect
Table 2 Genotype Distributions of FKBP5 SNPs in Children

\begin{tabular}{|c|c|c|c|c|c|c|c|}
\hline & A & $\mathbf{a}$ & $N_{\text {AA }}$ & $N_{\mathbf{A a}}$ & $N_{\mathbf{a a}}$ & MAF & $P_{\text {HWP }}$ \\
\hline rs|0807|5| & A & G & 91 & 64 & 6 & 0.236 & 0.194 \\
\hline rs3800373 & A & C & 76 & 72 & 13 & 0.304 & 0.476 \\
\hline rs7757037 & A & G & 49 & 89 & 23 & 0.419 & 0.086 \\
\hline rs9296158 & G & A & 73 & 74 & 14 & 0.317 & 0.433 \\
\hline rs3777747 & G & A & 49 & 88 & 24 & 0.422 & 0.127 \\
\hline rs6926133 & C & A & 89 & 64 & 8 & 0.248 & 0.413 \\
\hline rs9380524 & C & A & 81 & 63 & 17 & 0.301 & 0.371 \\
\hline rs| 6879378 & A & C & 159 & 2 & 0 & 0.006 & 0.937 \\
\hline rs 1475774 & G & A & $|5|$ & 10 & 0 & 0.031 & 0.684 \\
\hline rs47/3904 & A & G & 78 & 70 & 13 & 0.298 & 0.622 \\
\hline rs9470080 & G & A & 70 & 73 & 18 & 0.339 & 0.875 \\
\hline rs9380526 & A & G & 69 & 74 & 18 & 0.342 & 0.782 \\
\hline rs 10456432 & A & G & 139 & 19 & 3 & 0.078 & 0.026 \\
\hline rs9380529 & G & A & 57 & 81 & 23 & 0.394 & 0.500 \\
\hline rs9394314 & A & G & 80 & 71 & 10 & 0.283 & 0.266 \\
\hline rs2766533 & G & A & 61 & 80 & 20 & 0.373 & 0.426 \\
\hline rs 12200498 & G & A & 131 & 29 & I & 0.096 & 0.656 \\
\hline rs2817032 & A & G & 77 & 70 & 14 & 0.304 & 0.734 \\
\hline rs7751693 & G & A & 140 & 21 & 0 & 0.065 & 0.376 \\
\hline
\end{tabular}

The second and third columns list the nucleotides of the major (A) and minor (a) alleles respectively. The fourth, fifth, and sixth columns show the number of subjects with major allele homozygotes $\left(N_{A A}\right)$, heterozygotes $\left(N_{A a}\right)$, and minor allele homozygotes $\left(N_{a a}\right)$, respectively. The seventh column shows minor allele frequency (MAF). The eighth column shows p-values suggesting that the 19 FKBP5 SNPs did not deviate from Hardy-Weinberg proportions after correction for multiple comparisons.

on the left hippocampal volume $(p=0.018)$ and a statistically significant effect on the right hippocampal volume $(p=0.002)$. Further traditional regression analysis revealed that 7 out of 17 FKBP5 SNPs with MAF >0.05 showed statistically significant individual interactive effects with antenatal maternal depressive symptoms on the right hippocampal volume after Bonferroni correction for multiple comparisons $(p<0.05 / 17=0.00294$; uncorrected $p$-values in Supplementary Table S2 in the Supplementary Material). We noticed that the genotyping arrays used in this study did not cover a popular SNP, rs1360780, mentioned in the Introduction. Nevertheless, rs3800373 genotyped in this study is in the same haplotype block as rs1360780 (Szczepankiewicz et al, 2014). The analysis for individual FKBP5 SNPs showed a significant interaction between rs3800373 and antenatal maternal depressive symptoms on right hippocampal volume after Bonferroni correction for multiple comparisons (see Supplementary Table S2 in the Supplementary Material). A similar finding would be expected for rs 1360780 .

In the post hoc analysis, a genetic risk score was calculated for individual neonates by summing the number of minor alleles across all 19 FKBP5 SNPs. Figure 1 illustrates a scatter plot of antenatal maternal depressive symptoms and right hippocampal volume in neonates with low and high genetic risk groups. Neonates with the genetic risk score lower than or equal to its median showed a positive association between antenatal maternal depressive symptoms and the right hippocampal volume after partialling out the covariance of gestational age on the MRI visit day, neonatal total brain 

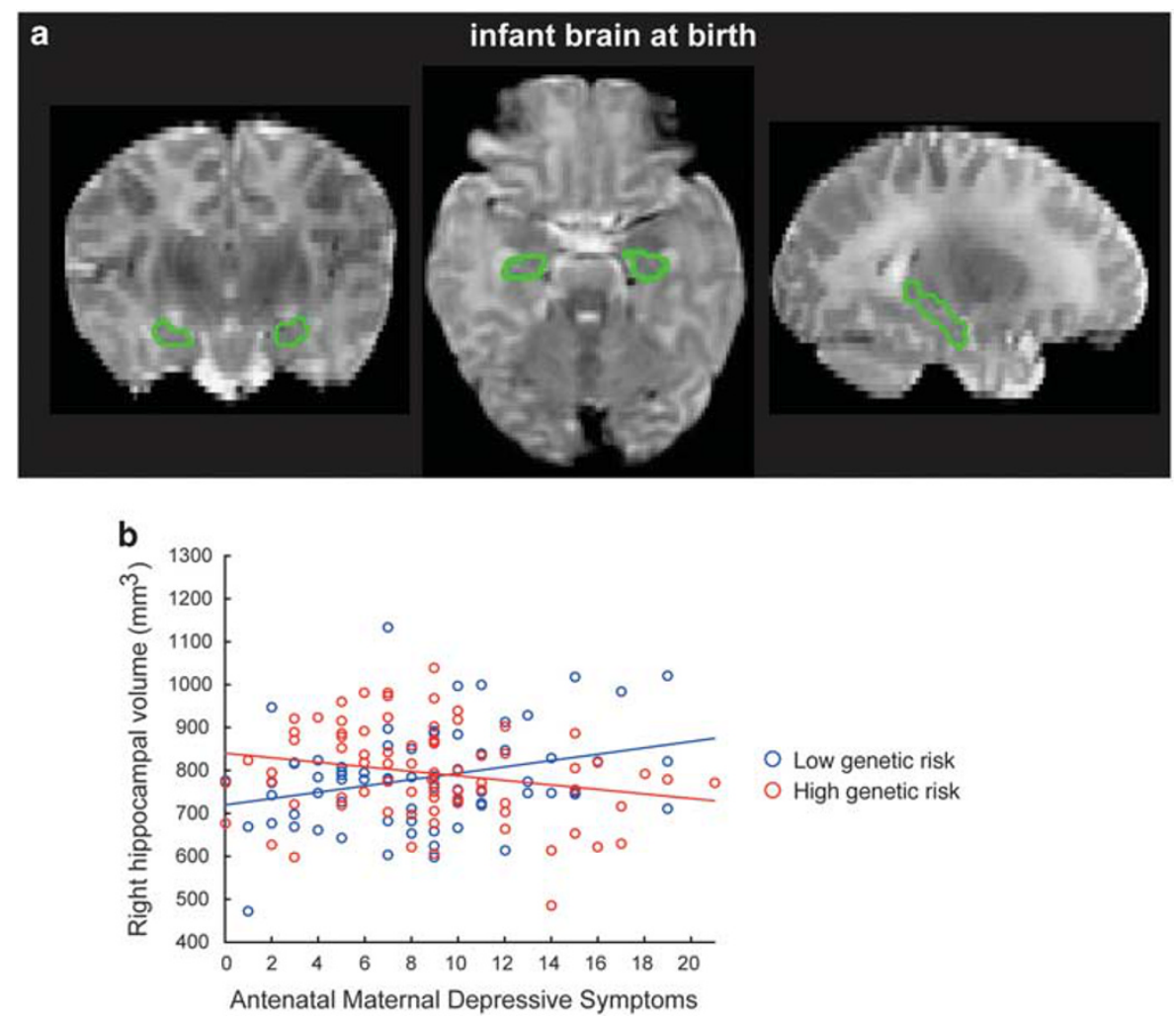

Figure I Hippocampus of neonatal brain. (a) Hippocampus on T2-weighted MRI. (b) Scatter plot for the relationship between right hippocampal volume and antenatal maternal depressive symptoms in neonates with a low FKBP5 genetic risk (blue) and a high FKBP5 genetic risk (red).

volume, maternal education, gender, and maternal ethnicity $\left(t_{80}=3.03, p=0.003\right)$. In contrast, neonates with the genetic risk score greater than its median showed a negative association between antenatal maternal depressive symptoms and the right hippocampal volume after partialling out the covariance of gestational age on the MRI visit day, neonatal total brain volume, maternal education, gender, and maternal ethnicity $\left(t_{77}=-2.00, p=0.049\right)$.

Similarly, our study revealed no significant interaction between FKBP5 and antenatal maternal depressive symptoms on bilateral amygdala volumes (left amygdala: $p=0.029$; right amygdala: $p=0.060$ ) and cortical thickness (corrected $p>0.05$ ).

\section{DISCUSSION}

Antenatal maternal depressive symptoms influence fetal neurodevelopment (Field et al, 2010). This study revealed that genetic variants in neonatal FKBP5 moderate the association of antenatal maternal depressive symptoms and neonatal right hippocampal morphology but show only marginal significance for the amygdala and cortical morphology. This effect was characterized by allelic variation in the neonatal FKBP5 gene and many of the neonatal FKBP5 SNPs (Supplementary Table S2 in the Supplementary Material). Our results suggest that the association between maternal depressive symptoms and in utero neurodevelopment of specific brain regions is modified through complex genetic variation in offspring FKBP5. Such genetic moderation was because of neonatal and not maternal genotype.
Thus, the FKBP5 moderation may explain, in part, the variation in neonatal phenotypic outcomes associated with antenatal maternal depressive symptoms. This conclusion is consistent with a wealth of studies showing that FKBP5 genotype moderates the impact of childhood adversity on neural development and function, as well as on mental health outcomes (Binder et al, 2008; Grabe et al, 2016; Tozzi et al, 2016; Xie et al, 2010).

FKBP5 genotype is associated with individual differences in hippocampal morphology and activation in response to threat (Fani et al, 2014; Holz et al, 2015). FKBP5 is highly expressed in the hippocampus (Scharf et al, 2011) that is strongly implicated in depression (Bremner et al, 2000; Campbell et al, 2004; Chen et al, 2010b; Sheline et al, 1996; Videbech and Ravnkilde, 2004) as well as with the vulnerability for depression in adolescents (Chen et al, 2010a). Stress reactivity predicts the risk for depression, and fMRI studies reveal that hippocampal activation, particularly in the right hemisphere (Pruessner et al, 2008), predicts individual differences in HPA response to stress (Pruessner et al, 2008). Our findings thus suggest a role for FKBP5 genotype in shaping fetal hippocampal development and subsequent differences in stress reactivity under conditions in increased antenatal maternal depressive symptoms. Such effects may underlie the observed increase in negative emotionality and stress reactivity that is apparent in the infant offspring of depressed mothers (Feldman et al, 2009).

Our study suggested that neonates with both risk factors (environmental and genetic) had a smaller right hippocampal volume than those with only the genetic risk factor. This 
is in line with the study in patients with major depression (Frodl et al, 2010). On the other hand, our study also suggested that neonates with only the environmental risk factor could have a larger hippocampal volume than those without any environmental or genetic risk factor. This may seem surprising. However, our study was based on a general population rather on a clinical sample. Antenatal maternal depressive symptoms were relatively mild among the mothers in this study. Sapolsky (2015) suggested that effects of stress could follow an inverted-U shape, where mild stress could be beneficial.

The strengths of this study include a unique opportunity to examine interactive effects of genetic variation and antenatal maternal depressive symptoms on neural development in neonate independent of postnatal influences, such as postnatal parenting and postnatal maternal mood (Chen et al, 2010b; Field et al, 2004; Fleming et al, 1988; Peterson and Weissman, 2011). Second, the study of the FKBP5 gene set is considered highly advantageous as the MixGE model incorporates the correlational nature of the underlying genetic variants. However, there were several limitations in this study. Antenatal maternal depressive symptoms were only assessed at one time point during pregnancy to minimize subject burden. The cohort was recruited during pregnancy, which resulted in the lack of maternal information before pregnancy. Though additional measurements may have allowed for a better understanding of specificity in timing, it is important to note that the second and third trimesters during pregnancy are critical periods when neural migration and synaptogenesis of the fetal brain rapidly develop. Moreover, our sample size for a genetic study is considered relatively moderate. This is mainly because of challenges in imaging neonates that is also the uniqueness of this study. Finally, our study did not consider linkage disequilibrium among the FKBP5 SNPs in the MixGE model that could result in the inflated statistical finding because of large haplotype blocks among these SNPs. Nevertheless, our study additionally performed regression analysis on the individual FKBP5 SNPs, independent of linkage disequilibrium among these SNPs, as complementary information to confirm our findings from the MixGE model.

In conclusion, these findings are the first to reveal that antenatal maternal depressive symptoms interact with genetic variants of neonatal FKBP5, resulting in alteration in the hippocampal morphology of neonates. Our results suggest that an increased risk for depression may be transmitted from mother to child during fetal life and that the effect is dependent upon neonatal FKBP5 genotype.

\section{FUNDING AND DISCLOSURE}

This research is supported by the Singapore National Research Foundation under its Translational and Clinical Research (TCR) Flagship Programme and administered by the Singapore Ministry of Health's National Medical Research Council (NMRC), Singapore- NMRC/TCR/004NUS/2008; NMRC/TCR/012-NUHS/2014; NMRC/CBRG/ $0039 / 2013$. Additional funding is provided by the Singapore Institute for Clinical Sciences, Agency for Science Technology and Research (A*STAR), Singapore. The authors declare no conflict of interest.

\section{REFERENCES}

Bai J, Abdul-Rahman MF, Rifkin-Graboi A, Chong Y-S, Kwek K, Saw SM et al (2012). Population differences in brain morphology and microstructure among Chinese, Malay, and Indian neonates. PLoS ONE 7: e47816.

Baumann C, Klauke B, Weber H, Domschke K, Zwanzger P, Pauli P et al (2013). The interaction of early life experiences with COMT val158met affects anxiety sensitivity. Genes Brain Behav 12: 821-829.

Binder EB, Bradley RG, Liu W, Epstein MP, Deveau TC, Mercer KB et al (2008). Association of FKBP5 polymorphisms and childhood abuse with risk of posttraumatic stress disorder symptoms in adults. JAMA 299: 1291-1305.

Binder EB, Salyakina D, Lichtner P, Wochnik GM, Ising M, Pütz B et al (2004). Polymorphisms in FKBP5 are associated with increased recurrence of depressive episodes and rapid response to antidepressant treatment. Nat Genet 36: 1319-1325.

Bremner JD, Narayan M, Anderson ER, Staib LH, Miller HL, Charney DS (2000). Hippocampal volume reduction in major depression. Am J Psychiatry 157: 115-118.

Campbell S, Marriott M, Nahmias C, MacQueen GM (2004). Lower hippocampal volume in patients suffering from depression: a meta-analysis. Am J Psychiatry 161: 598-607.

Chen MC, Hamilton JP, Gotlib IH (2010a). Decreased hippocampal volume in healthy girls at risk of depression. Arch Gen Psychiatry 67: 270-276.

Chen MC, Hamilton JP, Gotlib IH (2010b). Decreased hippocampal volume in healthy girls at risk of depression. Arch Gen Psychiatry 67: 270-276.

Cox JL, Holden JM, Sagovsky R (1987). Detection of postnatal depression. Development of the 10-item Edinburgh Postnatal Depression Scale. Br J Psychiatry 150: 782-786.

Fani N, King TZ, Reiser E, Binder EB, Jovanovic T, Bradley B et al (2014). FKBP5 genotype and structural integrity of the posterior cingulum. Neuropsychopharmacology 39: 1206-1213.

Feldman R, Granat A, Pariente C, Kanety H, Kuint J, GilboaSchechtman E (2009). Maternal depression and anxiety across the postpartum year and infant social engagement, fear regulation, and stress reactivity. J Am Acad Child Adolesc Psychiatry 48: 919-927.

Field T, Diego M, Dieter J, Hernandez-Reif M, Schanberg S, Kuhn C et al (2004). Prenatal depression effects on the fetus and the newborn. Infant Behav Dev 27: 216-229.

Field T, Diego M, Hernandez-Reif M (2010). Prenatal depression effects and interventions: a review. Infant Behav Dev 33: 409-418.

Fleming AS, Ruble DN, Flett GL, Shaul DL (1988). Postpartum adjustment in first-time mothers: Relations between mood, maternal attitudes, and mother-infant interactions. Dev Psychol 24: 71.

Frodl T, Reinhold E, Koutsouleris N, Donohoe G, Bondy B, Reiser $M$ et al (2010). Childhood stress, serotonin transporter gene and brain structures in major depression. Neuropsychopharmacology 35: 1383-1390.

Grabe HJ, Wittfeld K, Van der Auwera S, Janowitz D, Hegenscheid $\mathrm{K}$, Habes $\mathrm{M}$ et al (2016). Effect of the interaction between childhood abuse and rs1360780 of the FKBP5 gene on gray matter volume in a general population sample. Hum Brain Mapp 37: 1602-1613.

Hammen C, Brennan PA (2003). Severity, chronicity, and timing of maternal depression and risk for adolescent offspring diagnoses in a community sample. Arch Gen Psychiatry 60: 253-258.

Holz NE, Buchmann AF, Boecker R, Blomeyer D, Baumeister S, Wolf I et al (2015). Role of FKBP5 in emotion processing: results on amygdala activity, connectivity and volume. Brain Struct Funct 220: $1355-1368$.

Jiang L, Willner D, Danoy P, Xu H, Brown MA (2013). Comparison of the performance of two commercial genome-wide association 
study genotyping platforms in Han Chinese samples. G3 (Bethesda) 3: 23-29.

Klein DN, Lewinsohn PM, Seeley JR, Rohde P (2001). A family study of major depressive disorder in a community sample of adolescents. Arch Gen Psychiatry 58: 13-20.

Koenen KC, Uddin M (2010). FKBP5 polymorphisms modify the effects of childhood trauma. Neuropsychopharmacology 35: 1623.

Lahti J, Ala-Mikkula H, Kajantie E, Haljas K, Eriksson JG, Räikkönen K (2016). Associations between self-reported and objectively recorded early life stress, FKBP5 polymorphisms, and depressive symptoms in midlife. Biol Psychiatry 80: 869-877.

Lee S, Abecasis GR, Boehnke M, Lin X (2014). Rare-variant association analysis: study designs and statistical tests. Am J Hum Genet 95: 5-23.

Lekman M, Laje G, Charney D, Rush AJ, Wilson AF, Sorant AJ et al (2008). The FKBP5-gene in depression and treatment response-an association study in the Sequenced Treatment Alternatives to Relieve Depression (STAR ${ }^{*}$ D) Cohort. Biol Psychiatry 63: 1103-1110.

Levitt NS, Lindsay RS, Holmes MC, Seckl JR (1996). Dexamethasone in the last week of pregnancy attenuates hippocampal glucocorticoid receptor gene expression and elevates blood pressure in the adult offspring in the rat. Neuroendocrinology 64: $412-418$.

Oliphant A, Barker DL, Stuelpnagel JR, Chee MS (2002). BeadArray technology: enabling an accurate, cost-effective approach to highthroughput genotyping. Biotechniques 32: 56-58.

Peterson BS, Weissman MM (2011). A brain-based endophenotype for major depressive disorder. Annu Rev Med 62: 461-474.

Provencal N, Binder EB (2015). The neurobiological effects of stress as contributors to psychiatric disorders: focus on epigenetics. Curr Opin Neurobiol 30: 31-37.

Pruessner JC, Dedovic K, Khalili-Mahani N, Engert V, Pruessner M, Buss C et al (2008). Deactivation of the limbic system during acute psychosocial stress: evidence from positron emission tomography and functional magnetic resonance imaging studies. Biol Psychiatry 63: 234-240.

Qiu A, Anh TT, Li Y, Chen H, Rifkin-Graboi A, Broekman BF et al (2015a). Prenatal maternal depression alters amygdala functional connectivity in 6-month-old infants. Transl Psychiatry 5: e508.

Qiu A, Rifkin-Graboi A, Chen H, Chong Y, Kwek K, Gluckman PD et al (2013). Maternal anxiety and infants' hippocampal development: timing matters. Transl Psychiatry 3: e306.

Qiu A, Tuan TA, Ong ML, Li Y, Chen H, Rifkin-Graboi A et al (2015b). COMT haplotypes modulate associations of antenatal maternal anxiety and neonatal cortical morphology. Am J Psychiatry 172: 163-172.

Rifkin-Graboi A, Bai J, Chen H, Hameed WB, Sim LW, Tint MT et al (2013). Prenatal maternal depression associates with microstructure of right amygdala in neonates at birth. Biol Psychiatry 74: 837-844.

Sapolsky RM (2015). Stress and the brain: individual variability and the inverted-U. Nat Neurosci 18: 1344-1346.

Scharf SH, Liebl C, Binder EB, Schmidt MV, Müller MB (2011). Expression and regulation of the Fkbp5 gene in the adult mouse brain. PLoS ONE 6: e16883.

Sheline YI, Wang PW, Gado MH, Csernansky JG, Vannier MW (1996). Hippocampal atrophy in recurrent major depression. Proc Natl Acad Sci USA 93: 3908-3913.

Soh SE, Lee SSM, Hoon SW, Tan MY, Goh A, Lee BW et al (2012). The methodology of the GUSTO cohort study: a novel approach in studying pediatric allergy. Asia Pac Allergy 2: 144-148.

Szczepankiewicz A, Leszczynska-Rodziewicz A, Pawlak J, Narozna B, Rajewska-Rager A, Wilkosc M et al (2014). FKBP5 polymorphism is associated with major depression but not with bipolar disorder. J Affect Disord 164: 33-37.

Tozzi L, Carballedo A, Wetterling F, McCarthy H, O'Keane V, Gill $M$ et al (2016). Single-nucleotide polymorphism of the FKBP5 gene and childhood maltreatment as predictors of structural changes in brain areas involved in emotional processing in depression. Neuropsychopharmacology 41: 487-497.

Velders FP, Kuningas M, Kumari M, Dekker MJ, Uitterlinden AG, Kirschbaum C et al (2011). Genetics of cortisol secretion and depressive symptoms: a candidate gene and genome wide association approach. Psychoneuroendocrinology 36: 1053-1061.

Videbech P, Ravnkilde B (2004). Hippocampal volume and depression: a meta-analysis of MRI studies. Am J Psychiatry 161: 1957-1966.

Wang C, Sun J, Guillaume B, Ge T, Hibar DP, Greenwood CMT et al (2017). A set-based mixed effect model for geneenvironment interaction and its application to neuroimaging phenotypes. Front Neurosci 11: 191.

Weissman MM, Wickramaratne P, Nomura Y, Warner V, Pilowsky D, Verdeli H (2006). Offspring of depressed parents: 20 years later. Am J Psychiatry 163: 1001-1008.

Wichers M, Myin-Germeys I, Jacobs N, Peeters F, Kenis G, Derom C et al (2007). Genetic risk of depression and stressinduced negative affect in daily life. $B r J$ Psychiatry 191: 218-223.

Xie P, Kranzler HR, Poling J, Stein MB, Anton RF, Farrer LA et al (2010). Interaction of FKBP5 with childhood adversity on risk for post-traumatic stress disorder. Neuropsychopharmacology 35: 1684-1692.

Zobel A, Schuhmacher A, Jessen F, Höfels S, von Widdern O, Metten $\mathrm{M}$ et al (2010). DNA sequence variants of the FKBP5 gene are associated with unipolar depression. Int J Neuropsychopharmacol 13: 649-660.

Supplementary Information accompanies the paper on the Neuropsychopharmacology website (http://www.nature.com/npp) 